\title{
Compaction Model for Highly Deformable Particle Assemblies
}

\author{
David Cantor@, ${ }^{1, *}$ Manuel Cárdenas-Barrantes, ${ }^{2, \dagger}$ Itthichai Preechawuttipong, ${ }^{1, \$}$ \\ Mathieu Renouf, ${ }^{2,8}$ and Emilien Azéma $\circledast^{2, \|}$ \\ ${ }^{1}$ Department of Mechanical Engineering, Chiang Mai University, 239 Huay Kaew Road, 50200 Chiang Mai, Thailand \\ ${ }^{2}$ LMGC, Université de Montpellier, CNRS, 34090 Montpellier, France
}

(Received 13 February 2020; revised manuscript received 9 April 2020; accepted 4 May 2020; published 20 May 2020)

\begin{abstract}
The compaction behavior of deformable grain assemblies beyond jamming remains bewildering, and existing models that seek to find the relationship between the confining pressure $P$ and solid fraction $\phi$ end up settling for empirical strategies or fitting parameters. Using a coupled discrete-finite element method, we analyze assemblies of highly deformable frictional grains under compression. We show that the solid fraction evolves nonlinearly from the jamming point and asymptotically tends to unity. Based on the micromechanical definition of the granular stress tensor, we develop a theoretical model, free from $a d$ hoc parameters, correctly mapping the evolution of $\phi$ with $P$. Our approach unveils the fundamental features of the compaction process arising from the joint evolution of grain connectivity and the behavior of single representative grains. This theoretical framework also allows us to deduce a bulk modulus equation showing an excellent agreement with our numerical data.
\end{abstract}

DOI: 10.1103/PhysRevLett.124.208003

Powders, clays, rubber particles, cells, bubbles, and gels are some examples of particulate matter whose individual body deformation is crucial for understanding their bulk and mechanical behavior. Such body distortions are often dismissed or simplified by considering either the rigid body kinematics as the primary source of deformation or standing on the small-strain framework. However, explicitly describing the particles' large strains is essential for the development of technological applications such as the inclusion of rubber in concrete and pavements, and even for better understanding the flow properties of blood cells.

We focus this work on the compaction behavior of deformable grain assemblies beyond jamming, a subject widely investigated, of major industrial and engineering significance, yet misunderstood. The compaction of deformable matter has been addressed in experiments using ceramic, metallic, and pharmaceutical powders [1-5], gels [6-8], rubberlike particles [9-12], and even blood cells [13]. More recently, developments of numerical approaches based on meshless methods [14-16], the discrete element method [17,18], or the coupled finite element-discrete element methods [11,19-24] have enabled the exploration of the physics of deformable granular media.

It is worth mentioning the work of Heckel [2] who, employing an analogy between the compaction process and chemical reactions, proposed a model to predict the relationship between pressure $P$ and solid fraction $\phi$ (i.e., the ratio between the volume of the particles $V_{s}$ over the volume of the container $V$ ) of the form $P \propto-\ln (1-\phi)$. Although this model yields fair predictions, it relies upon two constants $(K$ and $B)$ not clearly related to physical or mechanical properties. Kim and Carroll [3] arrived at a similar relationship while studying metallic powders, but also introducing three constants yet to be defined by experiments and data fitting $\left(Y_{0}, \mu\right.$, and $\left.\gamma\right)$. Nezamabadi et al. [16] also introduced a model of the form $P \propto$ $-\phi \ln (\phi)$ employing an incremental formulation of the mean pressure and the macroscopic effective $P$-wave modulus, but standing, nonetheless, upon fitting constants $\left(c_{1}\right.$ and $\left.c_{2}\right)$. Finally, it is also notable that Zhang et al. [25], using a multiparticle finite element method, nicely reproduced the compaction curve after fitting a double logarithmic equation on their data. However, the origin of such a relation is not identified. Many other approaches can be found in the literature [5,26-33], though none of them is entirely satisfactory.

We can summarize the limitations of current models by the use of nonphysical parameters, the lack of accuracy at extreme pressures, and the missing physical derivation of the compaction equations. This situation calls for further investigation into the fundamental laws behind deformable granular media. But such a venture should admittedly rely on a proper description of the compaction process while taking into account the multicontact nature of the assembly and the particles' deformability.

In this Letter, we analyze the compaction of two-dimensional highly deformable particles for different coefficients of friction using a coupled discrete element and finite element method. We introduce an analytical model for the compaction behavior accounting for the particle connectivity, the applied pressure, and the solid fraction. Our model, free of ad hoc parameters, accurately predicts the sample density ranging from the granular jamming point, up to solids fractions close to the unity for any interparticle 
coefficient of friction. The bulk modulus evolution is also deduced from our approach.

We simulated assemblies of incompressible deformable particles undergoing isotropic compression in the frame of the contacts dynamic and finite element methods. This numerical coupling, also known as nonsmooth contact dynamics (NSCD) [34,35], considers unilateral contact conditions and dry friction between particles previously discretized in finite elements. Contact forces and body deformations are then systematically solved within a timestepping scheme. For the deformability of the grains, we used a hyperelastic constitute law following the neoHookean incompressible material model under plane strain conditions $[11,36]$. Note that the NSCD approach does not introduce regularization coefficients for the contact laws as other discrete element approaches do, making this numerical scheme robust and stable. Finally, we used an implementation of the NSCD on the free, open-source simulation platform LMGC90 [37]. For more details about the implementation of the numerical method, please see Refs. [34,38].

Numerical samples were built by depositing $N_{p}=1500$ disks into boxes using an algorithm based on simple geometrical criteria [39]. The disks, meshed using 92 triangular similar-size finite elements, presented a slight size dispersion around an average diameter $d$ to avoid crystallization. The choice of the mesh resolution is discussed later in Fig. 5. After deposition, the disks are initially found in a relatively dense configuration, yet not in contact. From this state, we set five different values of interparticle coefficient of friction $\mu$ in the interval $[0,0.8]$, in steps of 0.2. The coefficient of friction particles-walls and gravity were set to zero in all cases. A set of loading steps were then undertaken targeting stable values of applied pressure and solid fraction. We considered that a stable state was reached, for a given pressure, once the variations of the solid fraction remained below $0.05 \%$. Note that the pressure was gradually applied, so the particle-toparticle interaction and the particles' bulk rapidly damped the kinetic energy and elastic waves had little influence on the particle reorganization. Figure 1 shows two snapshots of stable configurations for the frictionless case at relative pressure $P / E$, with $E$ the Young modulus. We remark that as $P / E$ tends to zero, we have the corresponding jammed state for the rigid assembly of particles. In the following, values computed for such a reference state are denoted with the subscript " 0 ".

Figure 2 shows the evolution of $\phi$ with $P / E$ for all the coefficients of friction tested. We also display some of the compaction models previously mentioned fitting our numerical results for the frictionless case. Note that, for the case $\mu=0$, the isotropic and oedometric loadings remain indistinct, as shown in Refs. [40,41]. We can observe that, although the current models may be considered acceptable to some extent, they miss some of the (a)

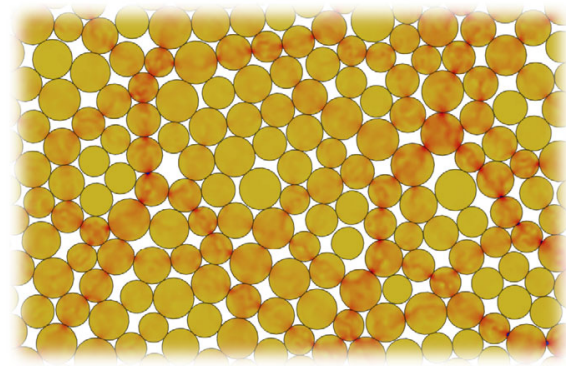

(b)

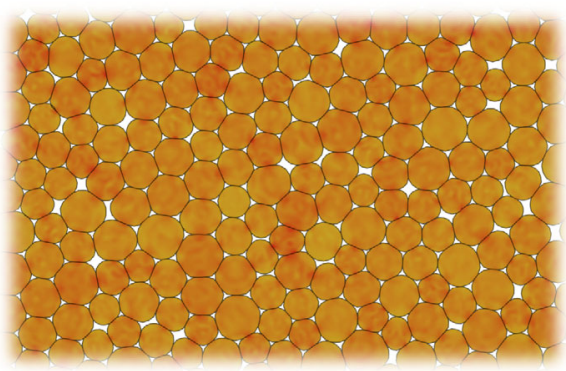

FIG. 1. Snapshots of the frictionless sample under a relative pressure (a) $P / E=5 \times 10^{-4}$ and (b) $P / E=5 \times 10^{-1}$. The color intensities are proportional to the volumetric deformation within the particles.

principal characteristics of the compaction curve. For instance, the data present two horizontal asymptotes: one for the perfectly rigid granular assembly $\left(\phi_{0}\right)$ and the other for extremely high pressures $\left(\phi_{\max }\right)$. The model of Kim and Carrol does not capture the first asymptote, while the model of Nezamabadi et al. does not capture the second. The models of Heckel and Zhang et al. display both asymptotes but slightly mismatch the evolution of the compaction curve for intermediate pressures. Recall as well that they rely on fitting parameters. We can also note that the coefficient of friction essentially modifies the maximal and minimal solid fraction since sliding mobilization is increasingly limited with $\mu[42,43]$.

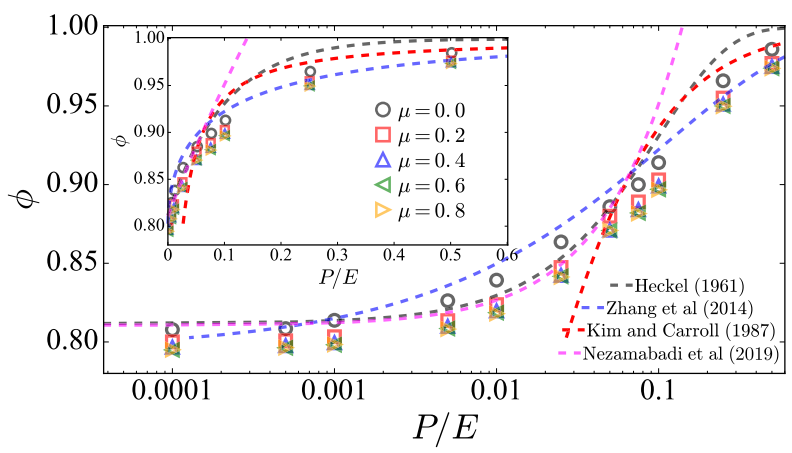

FIG. 2. Evolution of solid fraction $\phi$ as a function of $P / E$ for different value of coefficient of friction. We present our data along with four models set to best fit the frictionless case. Linear scale is shown in the inset. 
Keeping in mind the discrete nature of these assemblies, an adequate compaction model should rely on the physical phenomena taking place at the particles and contacts scales. Let us then consider the granular stress tensor written as $\sigma_{i j}=n_{c}\left\langle f_{i}^{c} \ell_{j}^{c}\right\rangle$ [44], with $n_{c}=N_{c} / V$ the density of contact number $N_{c}$ in the volume $V$ enclosed by the rigid walls, $\langle\bullet\rangle$ the average over the contact forces $f^{c}$ and the branch vectors $\ell^{c}$ (i.e., the vectors joining the centroids of the particles in contact). Then, we can compute the average pressure on the sample as $P_{\sigma}=\left(\sigma_{1}+\sigma_{2}\right) / 2$, with $\sigma_{1}$ and $\sigma_{2}$ the principal stresses of $\sigma_{i j}$. It is evident that $P=P_{\sigma}$.

It is also possible to express the contact density as $n_{c}=2 \phi Z / \pi d^{2}$, considering a small particle size dispersion around diameter $d$, and with the coordination number $Z=2 N_{c} / N_{p}$. These definitions let us rewrite the stress tensor as $\sigma_{i j}=\left(2 \phi Z / \pi d^{2}\right)\left\langle f_{i}^{c} \ell_{j}^{c}\right\rangle$. And, finally, taking into account the definition of $P$ via the principal stresses of $\sigma_{i j}$, we can deduce a microstructural equation of the mean pressure as [45-47]

$$
P=\frac{\phi Z}{\pi} \sigma_{\ell}
$$

with $\sigma_{\ell}=\left\langle f^{c}\right\rangle\left\langle\ell^{c}\right\rangle / d^{2}$, a measure of the interparticle stresses taking into account that contact forces and branches are not correlated. Equation (1) reveals the joint relation between $P$ and $\phi$ and the granular microstructure in terms of particle connectivity and force transmission. Note that the connectivity $Z$ of an assembly of deformable grains is closely linked to the evolution of solid fraction as denser particle configurations lead to higher coordination numbers. Additionally, it has been systematically reported in the literature that the relation $Z-\phi$ obeys a power law of the form $\left(Z-Z_{0}\right)=k\left(\phi-\phi_{0}\right)^{\alpha}$, with exponent $\alpha \simeq 0.5$ $[11,16,44]$, and $k \simeq 5.1$ a structural parameter fully defined as the $P / E \rightarrow \infty$, and $\phi$ and $Z$ reach maximal values. We found the same proportionality in our experiments independently of the coefficient of friction (see Fig. 3).

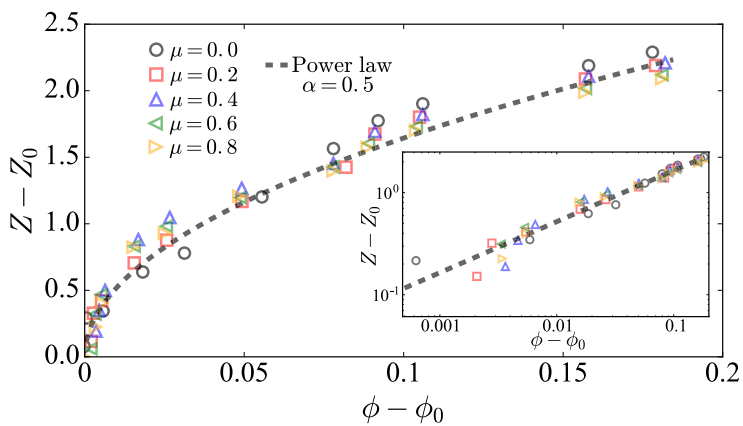

FIG. 3. The reduced coordination number $Z-Z_{0}$ as a function of the reduced solid fraction $\phi-\phi_{0}$ for all the coefficient of friction tested (log-log representation in the inset). The dashed black line displays the power law relation $Z-Z_{0}=k\left(\phi-\phi_{0}\right)^{\alpha}$.
Alternatively, we can assume that the packing of particles roughly behaves like a continuum medium and, thus, the mean compressive stress $P$ can be related to the macroscopic cumulative volumetric strain $\varepsilon_{v}=-\ln \left(\phi_{0} / \phi\right)$ using the bulk modulus $K(\phi)=$ $(d P / d \phi)\left(d \phi / d \varepsilon_{v}\right)$. Assuming homogeneous strains within the sample, we can replace the discrete system by an assembly of springs of average length $\left\langle\ell^{c}\right\rangle$ [47]. Then, we can define a local volumetric strain as $\varepsilon_{v, \ell}=$ $2 \ln \left(\left\langle\ell^{c}\right\rangle / d\right)$. Our numerical simulations also revealed that $\varepsilon_{v}=2 \varepsilon_{v, \ell}$. Using the above expressions, the power law between $Z$ and $\phi$, and assuming $\sigma_{\ell}=E \varepsilon_{v, \ell}$, we can obtain a first prediction of the bulk modulus employing the derivative of Eq. (1) and the first-order Taylor expansion of $\varepsilon_{v}$ as

$$
K_{1}(\phi) / E=\frac{Z \phi}{\pi}\left(\frac{5}{4}-\frac{\phi_{0}}{2 \phi}\right)-\frac{Z_{0} \phi}{4 \pi}
$$

This equation is reminiscent of those given in Refs. [4451] deduced upon a small-strain framework (i.e., HertzMindlin contact law) and the effective medium theory. Figure 4 shows the evolution of the bulk modulus measured in our simulations and that computed using Eq. (2), both as a function of $\phi$. As also noted in previous studies, fair predictions of bulk modulus result for the small-strain domain when standing on the equivalent medium approach. Yet, we observe an increasing mismatch as the solid fraction tends to unity since the assembly of grains starts to behave as a nondeformable solid (i.e., the corresponding bulk modulus diverges). This observation suggests that defining local strains only by means of the contact deflections (i.e., $\sigma_{l}=E \varepsilon_{v, \ell}$ ) should be reconsidered.

An alternative approach consists of building the macroscopic strain in terms of local contributions of Voronoi cells enveloping each particle as $\varepsilon_{c, \ell}=-\ln \left(V_{\ell} / V_{\ell, 0}\right)$, with $V_{\ell}$ the volume of each Voronoi cell. Note that by construction, $\varepsilon_{v}=\varepsilon_{c, \ell}$. The question rising from this approach is to what

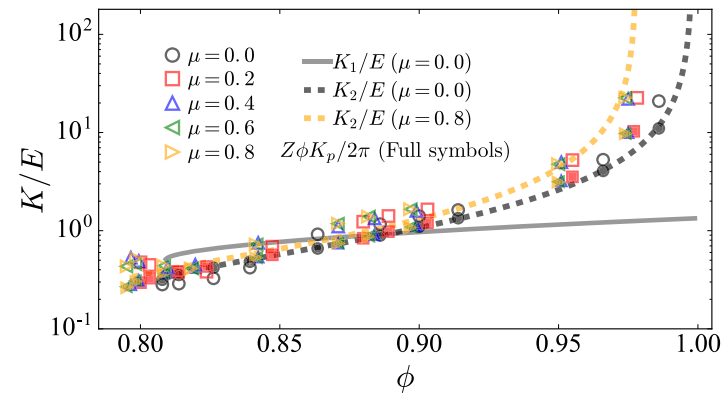

FIG. 4. Measured modulus $K$ normalized by $E$ (empty symbols) along with its microstructural origin proposed in Eq. (3) (full symbols). The gray line is the prediction using Eq. (2) for $\mu=0$, and dashed black and orange lines are predictions using Eq. (7) for $\mu=0$ and $\mu=0.8$, respectively. 


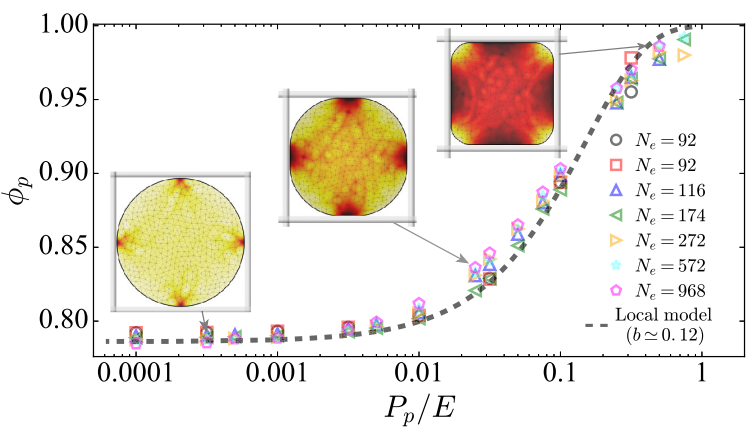

FIG. 5. Compaction curve of a single particle for different mesh resolutions. Red squares $\left(P_{p}\right.$ fixed and $\mathrm{E}$ varied) and black circles ( $E$ fixed and $P_{p}$ varied) are tests on a particle with 92 finite elements. For the other mesh resolutions, $E$ was fixed and $P_{p}$ varied. Snapshots for $N_{e}=968$ display the volumetric strain at different moments of the compression.

extent can the volumetric behavior of such a local configuration be related to the bulk modulus of the multiparticle system? We can address this issue by considering an analogous elementary system composed of a single particle between four rigid walls following the multiparticle boundary conditions (see insets of Fig. 5).

Figure 5 shows the evolution of the solid fraction $\phi_{p}$ and applied pressure $P_{p}$ for the single-particle case after applying the same strain increments as for the multiparticle system (i.e., $\varepsilon_{v} \equiv \varepsilon_{v, p}$, with the latter the volumetric strain for the single-particle compression). We observe that the single-particle compression is roughly akin to the multiparticle trend, supporting the idea of a deep relation between the two systems. We also tested a different number of finite elements $N_{e}$, corroborating the fact that the mesh resolution, at least for the element densities tested, has no major effect on the results. We then computed the bulk modulus for the single-particle compression as $K_{p}\left(\phi_{p}\right)=$ $\left(d P_{p} / d \phi_{p}\right)\left(d \phi_{p} / d \varepsilon_{v, p}\right)$, with $\varepsilon_{v, p}=-\ln \left(\phi_{p, 0} / \phi_{p}\right)$, and $\phi_{p, 0}=\pi / 4$ the solid fraction as $P_{p} / E$ tends to zero.

Figure 4 also shows that the macroscopic bulk modulus of the assembly $K$ increases as the relation $(Z \phi / 2 \pi)$ does, allowing us to reinterpret the micromechanical origin of the bulk modulus as

$$
K \equiv\left(\frac{Z \phi}{2 \pi}\right) K_{p}+\mathcal{O}
$$

with $\mathcal{O}$ negligible high-order terms. Equation (3) suggests that the single-particle configuration is no other than the smallest representative scale in our multiparticle system. Supported on Eq. (3), we can also reinterpret Eq. (1) as

$$
P \equiv\left(\frac{Z \phi}{2 \pi}\right) P_{p}
$$

where we can deduce that the $\mathcal{O}$ in Eq. (3) is simply related to the derivatives $\left(d Z \phi P_{p}+Z d \phi P_{p}\right)$. Equations (3) and (4) reveal that the elastic and compaction properties of a deformable particle assembly are scalable from the behavior of an individual particle. More precisely, a description of a single-particle compression should be a central ingredient for the description of the behavior of the assembly.

We remark that the compaction behavior of a single particle can be easily described as

$$
P_{p} / E=-b \ln \left(\frac{\phi_{p, \max }-\phi_{p}}{\phi_{p, \max }-\phi_{p, 0}}\right)
$$

with $\phi_{p, \max }$ the maximal solid fraction attainable and $b$ a constant of proportionality found to be $\simeq 0.12$. This relation is obtained from the corresponding loss of void space analogous to the collapse of a cavity within an elastic medium under isotropic compression [52]. Equation (5) is displayed in Fig. 5 using a black dashed line.

Finally, introducing Eq. (5) and the relation between $Z$ and $\phi$ into Eq. (4), and noting that $\phi$ can be mapped to $\phi_{p}$ (since $\varepsilon_{v} \equiv \varepsilon_{p, v}$ ), we get the compaction equation

$$
P / E=-\frac{b \phi}{2 \pi}\left\{Z_{0}+k\left(\phi-\phi_{0}\right)^{\alpha}\right\} \ln \left(\frac{\phi_{\max }-\phi}{\phi_{\max }-\phi_{0}}\right) .
$$

Figure 6 presents our numerical data and the compaction equation for the cases $\mu=0$ and $\mu=0.8$. As we can observe, the predictions given by Eq. (6) are outstanding for any pressure capturing the asymptotes for vanishing and extremely high pressures and the effect of the coefficient of friction. Our compaction equation also allows us to predict the saturation of solid fraction as $\mu$ increases. Simple fitting of Eq. (6) reveals that $\phi_{\max }=0.998$ for the frictionless case, while $\phi_{\max }=0.968$ for $\mu=0.8$.

Going one step further, derivating Eq. (6) and neglecting high-order terms (e.g., in the form of $\phi^{\alpha+1} \ln \phi$ ), we get a second expression for the bulk modulus evolution as

$$
K_{2}(\phi) / E \simeq \frac{b \phi^{2}}{2 \pi\left(\phi_{\max }-\phi\right)}\left\{Z_{0}+k\left(\phi-\phi_{0}\right)^{\alpha}\right\} .
$$

Finally, Fig. 4 presents the above relation perfectly reproducing the evolution of $K$ throughout the deformation for the cases $\mu=0$ and $\mu=0.8$. Note, however, that the coefficient of friction has little influence on the macroscopic bulk modulus for the small deformation domain, but then its effects increase for larger strains. Such a highquality capacity of prediction also supports, a posteriori, the validity of our analysis.

In summary, we developed a compaction model whose ingredients are deeply encoded in the evolution of grain connectivity and the bulk behavior of a representative single particle. Our systematic approach was capable of 


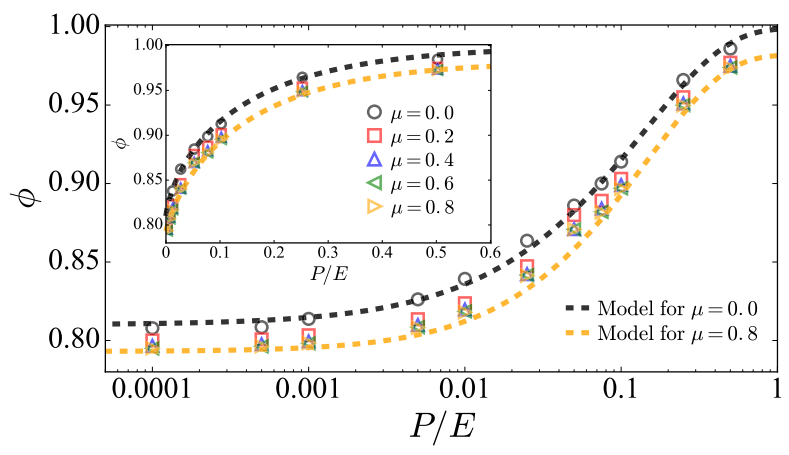

FIG. 6. Compaction curve (same as Fig. 2) along with Eq. (6) for $\mu=0$ (black dashed line) and $\mu=0.8$ (orange dashed line). The inset presents the data in linear scale.

producing a compaction equation free of $a d$ hoc parameters and stands on well-defined quantities (i.e., solid fraction, coordination number, and applied pressure). Although our compaction model is deduced in the frame of the neoHookean material model, our purpose is also to present a methodical and micromechanically based approach for the analysis of deformable particle assemblies under compression. Indeed, this approach could be generalized to different material models (elastic, hyperelastic, plastic, etc.) or to other loading configurations such as the oedometric compression test. These alternative mechanical considerations may lead to distinct functional forms, other than logarithmic, but certainty linked to the behavior of individual particles. It will also be of great interest to extend this work using different grain shapes, to consider mixtures of bodies with varying elastic properties, and to characterize the shear behavior.

We thank Jonathan Barés for fruitful discussions. We acknowledge the support of the High-Performance Computing Platform MESO@LR. D.C. gratefully acknowledges the support provided by Chiang Mai University through a postdoctoral fellowship. We also thank Franny McGill for her helpful advice and support.

*david.cantor@cmu.ac.th

†manuel-antonio.cardenas-barrantes@umontpellier.fr

*itthichai.p@cmu.ac.th

§mathieu.renouf@umontpellier.fr

"emilien.azema@umontpellier.fr

[1] A. Cooper and L. Eaton, J. Am. Ceram. Soc. 45, 97 (1962).

[2] R. W. Heckel, Trans. Metall. Soc. AIME 221, 671 (1961).

[3] K. Kim and M. Carroll, Int. J. Plast. 3, 63 (1987).

[4] K. Kawakita and K. H. Lüdde, Powder Technol. 4, 61 (1971).

[5] I. Wünsch, J. H. Finke, E. John, M. Juhnke, and A. Kwade, Pharmaceutics 11, 1 (2019).

[6] M. Guo and H. M. Wyss, Macromol. Mater. Eng. 296, 223 (2011).
[7] J. A. Dijksman, N. Brodu, and R. P. Behringer, Rev. Sci. Instrum. 88. 051807 (2017).

[8] R. Stannarius, D. Sancho Martinez, T. Finger, E. Somfai, and T. Börzsönyi, Granular Matter 21, 56 (2019).

[9] Y. L. Lin, D. M. Wang, W. M. Lu, Y. S. Lin, and K. L. Tung, Chem. Eng. Sci. 63, 195 (2008).

[10] A. Platzer, S. Rouhanifar, P. Richard, B. Cazacliu, and E. Ibraim, Granular Matter 20 (2018).

[11] T.-L. Vu, J. Barés, S. Mora, and S. Nezamabadi, Phys. Rev. E 99, 062903 (2019).

[12] T.-L. Vu and J. Barés, Phys. Rev. E 100, 042907 (2019).

[13] J. Mauer, S. Mendez, L. Lanotte, F. Nicoud, M. Abkarian, G. Gompper, and D. A. Fedosov, Phys. Rev. Lett. 121, 118103 (2018).

[14] A. Boromand, A. Signoriello, F. Ye, C. S. O’Hern, and M. D. Shattuck, Phys. Rev. Lett. 121, 248003 (2018).

[15] A. Boromand, A. Signoriello, J. Lowensohn, C. S. Orellana, E. R. Weeks, F. Ye, M. D. Shattuck, and C. S. O'Hern, Soft Matter 15, 5854 (2019).

[16] S. Nezamabadi, X. Frank, J. Y. Delenne, J. Averseng, and F. Radjai, Comput. Phys. Commun. 237, 17 (2019).

[17] A. Hassanpour and M. Ghadiri, Powder Technol. 141, 251 (2004).

[18] N. Brodu, J. A. Dijksman, and R. P. Behringer, Phys. Rev. E 91, 032201 (2015).

[19] A. T. Procopio and A. Zavaliangos, J. Mech. Phys. Solids 53, 1523 (2005).

[20] F. Güner, Ö. N. Cora, and H. Sofuoğlu, Powder Technol. 271, 238 (2015).

[21] N. Abdelmoula, B. Harthong, D. Imbault, and P. Dorémus, J. Mech. Phys. Solids 10-9, 142 (2017).

[22] F. Huang, X. An, Y. Zhang, and A. B. Yu, Powder Technol. 314, 39 (2017).

[23] G. Mollon, Granular Matter 20, 39 (2018).

[24] P. Loidolt, M. H. Ulz, and J. Khinast, Powder Technol. 336, 426 (2018).

[25] Y. X. Zhang, X. Z. An, and Y. L. Zhang, Appl. Phys. A 118, 1015 (2015).

[26] J. van der Zwan and C. A. Siskens, Powder Technol. 33, 43 (1982).

[27] H. Leuenberger, Int. J. Pharm. 12, 41 (1982).

[28] V. Tvergaard, in Adv. Appl. Mech. (Elsevier, 1989), Vol. 27, pp. 83-151.

[29] R. Panelli and F. Ambrozio Filho, Powder Technol. 114, 255 (2001).

[30] P. J. Denny, Powder Technol. 127, 162 (2002).

[31] T. Çomoğlu, Ankara Univ. Eczacilik Fak. Derg. 36, 123 (2007).

[32] J. M. Montes, F. G. Cuevas, J. Cintas, and Y. Torres, Granular Matter 12, 617 (2010).

[33] L. Parilak, E. Dudrova, R. Bidulsky, and M. Kabatova, Powder Technol. 322, 447 (2017).

[34] M. Jean, Comput. Methods Appl. Mech. Eng. 177, 235 (1999).

[35] M. Renouf, F. Dubois, and P. Alart, J. Comput. Appl. Math. 168, 375 (2004).

[36] R. S. Rivlin, Phil. Trans. R. Soc. A 240, 459 (1948).

[37] https://git-xen.lmgc.univ-montp2.fr/lmgc90/lmgc90_user/ wikis/home. 
[38] F. Dubois, V. Acary, and M. Jean, C.R. Mec. 346, 247 (2018).

[39] W. M. Visscher and M. Bolsterli, Nature (London) 239, 504 (1972).

[40] P. E. Peyneau and J. N. Roux, Phys. Rev. E 78, 041307 (2008).

[41] M. H. Khalili, J. N. Roux, J. M. Pereira, S. Brisard, and M. Bornert, Phys. Rev. E 95, 032907 (2017).

[42] M. van Hecke J. Condens. Matter Phys. 22, 033101 (2010).

[43] L. E. Silbert, D. Ertaş, G. S. Grest, T. C. Hasley, and D. Levine, Phys. Rev. E 65, 031304 (2002).

[44] B. Andreotti, Y. Forterre, and O. Pouliquen, Granular Media: Between Fluid and Solid (Cambridge University Press, Cambridge, England, 2013).
[45] I. Agnolin and J. N. Roux, Phys. Rev. E 76, 061304 (2007).

[46] M. H. Khalili, J. N. Roux, J. M. Pereira, S. Brisard, and M. Bornert, Phys. Rev. E 95, 032908 (2017).

[47] R. J. Bathurst and L. Rothenburg, J. Appl. Mech. Trans. ASME 55, 17 (1988).

[48] K. Walton, J. Mech. Phys. Solids 35, 213 (1987).

[49] J. D. Goddard, Proc. R. Soc. A 430, 105 (1990).

[50] H. A. Makse, N. Gland, D. L. Johnson, and L. M. Schwartz, Phys. Rev. Lett. 83, 5070 (1999).

[51] L. La Ragione and V. Magnanimo, Phys. Rev. E 85, 031304 (2012).

[52] M. M. Carroll and K. T. Kim, Powder Metall. 27, 153 (1984). 